\title{
Autosomal dominant polycystic liver disease: a second family
}

\author{
Gabriel Berrebi, ${ }^{1}$ Robert P. Erickson, ${ }^{2}$ and Byron W. Marks ${ }^{3}$ \\ Departments of Medicine, ${ }^{1}$ Human Genetics, ${ }^{2}$ and Radiology ${ }^{3}$, University of Michigan Medical School, \\ Ann Arbor, Michigan, U.S.A.
}

\begin{abstract}
An autosomal dominant pattern of transmission has been established for polycystic kidney disease. The degree of cystic involvement of other organs has been variable. The genetic pattern of transmission of polycystic liver disease independent of cystic kidney disease has never been established. We present a second family with polycystic liver disease without kidney disease. The lack of renal cysts is unlikely to be due to variable expressivity and penetrance of the gene for polycystic kidney disease. The liver cysts may be of late onset since none of the proband's four children demonstrate cysts. Alternatively, none of these four individuals may have received the gene for polycystic liver disease from their affected mother. The family described supports an autosomal dominant pattern of inheritance for polycystic liver disease.
\end{abstract}

Received 18 February, accepted for publication 16 April 1982

Key words: Abdominal computerized axial tomography; abdominal ultrasound ; autosomal dominant inheritance; kidney cysts; liver cysts.

An autosomal dominant pattern of transmission has been established for polycystic kidney disease (Cairns 1925). The age of onset of clinical manifestations as well as the degree of cystic involvement of other organs has been variable. Williams \& Price (1970) reported the incidence of cystic livers in polycystic kidney disease patients to be $75 \%$ while Luzzatto (cited in Moschowitz 1906) reported the lowest incidence of this association at $5.5 \%$. Conversely, the reported frequency of polycystic kidney disease in patients with cystic livers has varied from an incidence of $88.2 \%$ (Moschowitz 1906) to 30.5\% (Davis 1937). Recently Sotaniemi et al. (1979) described a family in which four of ten offspring of a mother with polycystic liver had cystic disease of the liver and no clinical renal abnormalities. We present a second family with polycystic liver disease without kidney disease and discuss its possible pattern of inheritance.

\section{Family History}

We studied members in three generations of the L family (pedigree in Fig. 1) to document the extent of cystic involvement of the liver and kidneys. None of the members of this family who were studied had any complaints of clinical kidney disease and/or a past medical history of kidney ailments. The only members who had documented liver cysts prior to our study were I-3 (aunt) and II-3 (sister), both of whom will be presented in detail below, in addition to our own patient, II-2. Cases III-1 through III-4 were asymptomatic and have remained asymptomatic for liver and kidney disease. The results of their ultrasound examinations will be reviewed. 


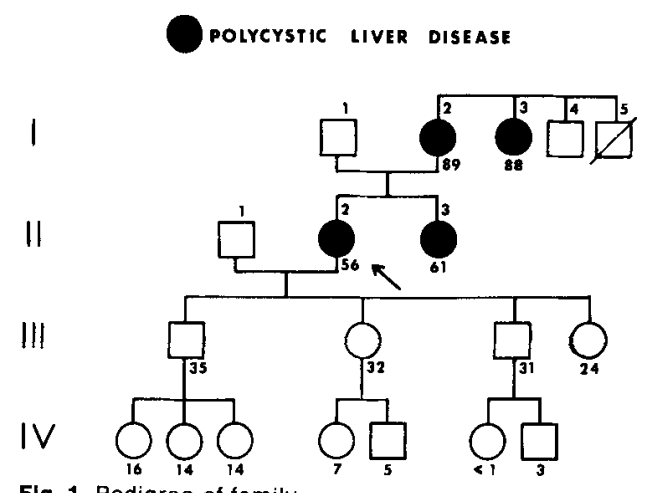

Flg. 1. Pedigree of family.

Case I-2, who has remained completely asymptomatic for clinical disease was studied by computerized axial tomography of the abdomen; the results will also be presented.

\section{Case II-2 (Proband)}

This patient is a 56-year-old female who first complained of right upper quadrant pain in 1972. She was diagnosed by liver scan and ultrasound as having cysts in her liver. She did well until 1 year prior to study when she complained of progressive right upper quadrant pain and underwent repeated ultrasonography which showed multiple liver cysts, the largest of which was $13 \mathrm{~cm}$ in diameter. The patient was also found to have a single cyst in the right kidney. There were no complaints of renal symptoms; the patient has had mild hypertension for approximately 20 years. To rule out other diseases which may have been contributing to the patient's symptoms, she underwent oral cholecystogram, upper gastrointestinal contrast study, barium enema and endoscopy, all of which showed no other causes for her physical complaints. Six months prior to study, the patient noticed increased right upper quadrant pain, whitish chalky stools, pruritus, dark urine and yellowing of her skin. Her serum bilirubin was $8.0 \mu \mathrm{g} / \mathrm{dl}$; alkaline phosphatase was $300 \mathrm{IU} / 1$; and serum glutamine oxamate transferase was $50 \mathrm{IU} / 1$. The patient improved clinically with only analgesic therapy and her

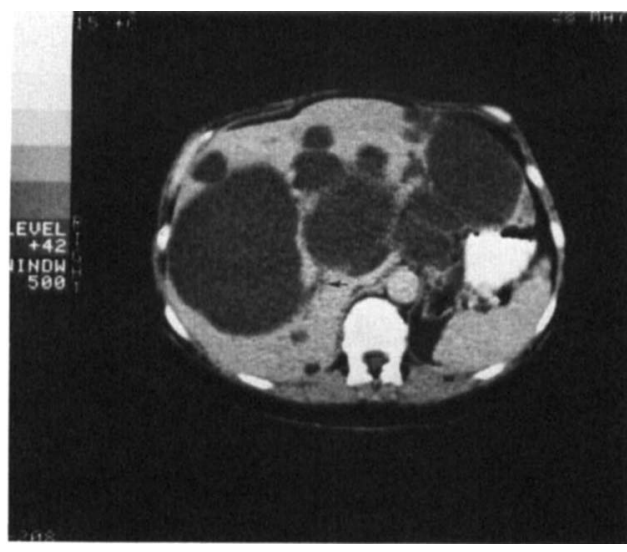

Fig. 2. Computerized axial tomography demonstrating multiple liver cysts in daughter (arrowhead marks dilated biliary ducts).

bilirubin returned to $4.0 \mu \mathrm{g} / \mathrm{dl}$; her alkaline phosphatase decreased to 281 IU/1 and her serum glutamine oxamate transferase stayed elevated at $60 \mathrm{IU} / 1$.

The first documentation of the patient's liver size occurred 5 months prior to study when it was noted to be $25 \mathrm{~cm}$ in length at the midclavicular line. Repeat ultrasound confirmed that the source of the hepatomegaly was polycystic liver disease; it also confirmed one right kidney cyst and found no ovarian cysts.

The patient underwent endoscopic retrograde cholangiopancreatography which showed normal hepatic and biliary tree without common bile duct or common hepatic duct abnormalities. No stones were visualized. The patient underwent computerized axial tomography on a GE8800 scanner with a high resolution detector assay. She was scanned at 1 $\mathrm{cm}$ intervals through the liver, kidney and pancreas. The scan revealed an enlarged liver with cysts too numerous to count, ranging in size from $0.5 \mathrm{~cm}$ to $16 \mathrm{~cm}$ in diameter (Fig. 2). While no segment of the liver was spared, the majority of the cysts were located in the superior portion of the right lobe and in the left lateral portion of the left lobe. Associated with the cysts were several areas of focal dilatation of the 


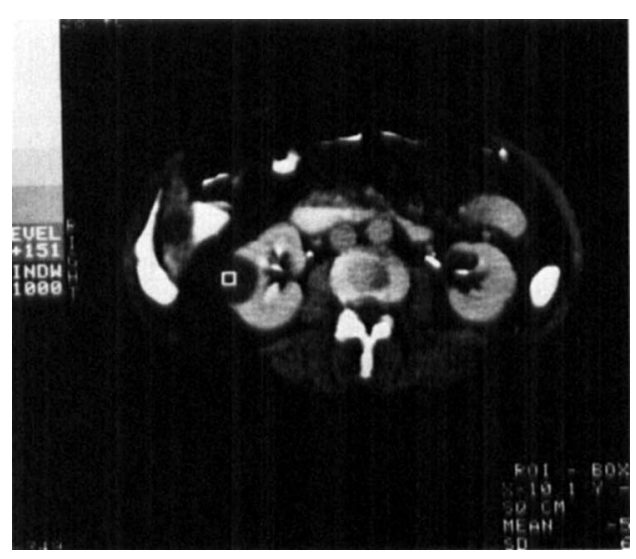

Fig. 3. Right renal cyst and hepatic cyst in Case II-2.

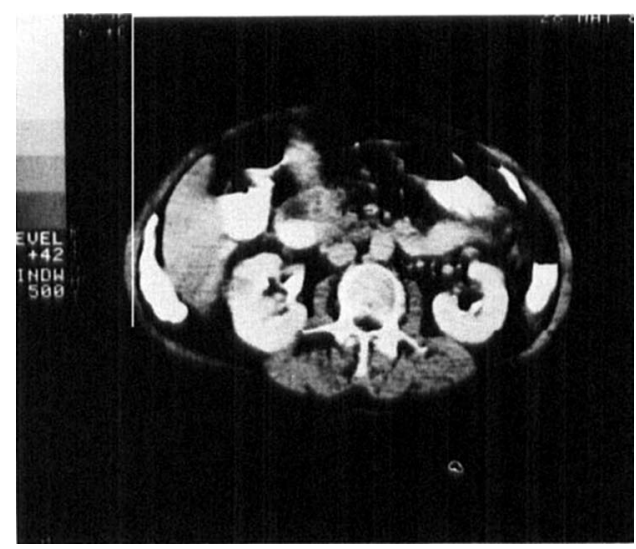

Fig. 4. Computerized axial tomography showing $2-3 \mathrm{~mm}$ cysts in head of pancreas in daughter (arrowheads).

bile ducts. There was a single cortical cyst on each of the patient's kidneys (Fig. 3). The kidneys were $11 \mathrm{~cm}$ on the left and $10 \mathrm{~cm}$ on the right. Numerous small $2-3 \mathrm{~mm}$ cysts were seen throughout the pancreas (Fig. 4). The pancreas was normal in size. No splenic cysts were noted.

Case I-2 (Mother: Age 89)

This elderly female has never had symptoms of liver and/or renal disease and therefore had never undergone any evaluations to assess cystic disease. As she is the mother of our patient, and since her sister (Case I-3) had documented liver cystic disease, we investigated this patient with computerized axial tomography of the abdomen. The liver, kidney and pancreas were scanned using contrast media. The scan showed six liver cysts, all $1 \mathrm{~cm}$ in diameter or less (Fig. 5). In this patient there was a distinct predilection for the subcapsular portion of the liver with four of six cysts being located within $0.5 \mathrm{~cm}$ of the liver capsule. There was a single $3 \mathrm{~cm}$ cortical cyst of the right kidney, while the left kidney contained two cortical and two sinus cysts (Fig. 6). The right kidney measured $10 \mathrm{~cm}$ and the left kidney measured $9 \mathrm{~cm}$. No

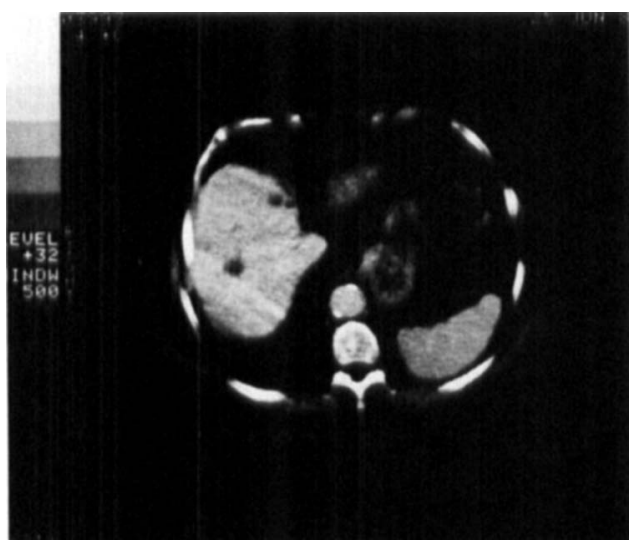

=lg. 5. Computerized axial tomography showing hepatic iysts in Case 1-2.

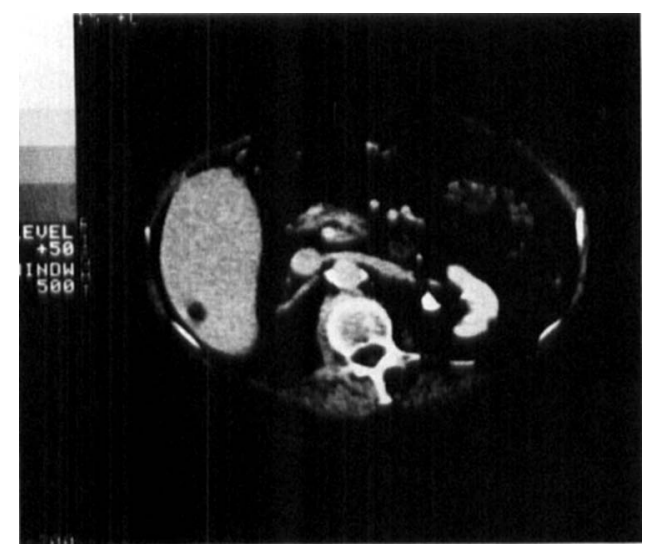

FIg. 6. Computerized axial tomography showing renal cysts in Case 1-2. 
pancreatic or splenic cysts were identified. She has remained asymptomatic.

Case 1-3 (Aunt; Age 88)

This female patient was in a good state of health until 12 years prior to our study when she was noticed to have gastrointestinal complaints. She underwent barium enema and upper gastrointestinal contrast studies that were within normal limits. At one outside hospital she underwent technetium sulfur colloid scans of her liver which showed a very large, central, rounded area of decreased concentration of the isotope. The scan also showed a few, much smaller, similar defects along the periphery of the liver. She underwent liver biopsy to rule out neoplasia, which showed proper orientation of portal spaces and central veins. There was no finding of neoplasia on the biopsy specimen. Eleven years prior to our study, the patient was taken to surgery for exploratory laparotomy. Her diagnosis after surgical exploration was polycystic liver disease with one visible cyst on the right kidney. As part of her evaluation, to rule out malignant disease, this patient underwent intravenous pyelography which showed prompt excretion and good concentration bilaterally. There were no filling defects, obvious mass, or calculi. It was noted that the right kidney appeared displaced downward by the enlarged liver. This patient has remained clinically free of liver symptoms and renal complaints. She has always had normal creatinine and blood urea nitrogen levels. Her serum bilirubin has also remained within normal limits.

\section{Case II-3 (Sister)}

This adult female, now aged 61 , noted abdominal enlargement at age 34 . The abdominal enlargement was associated with right upper quadrant abdominal pain, sharp in character. Exploratory laparotomy 25 years prior to our study showed a cystic liver; biopsy confirmed the diagnosis of polycystic liver disease. Her discomfort continued and 17 years prior to our study the patient underwent percutaneous needle drainage with a total of approximately $5000 \mathrm{cc}$ of fluid removed. Her intravenous pyelogram was consistently negative for renal cysts. Because of worsening abdominal pain and elevation of liver functions, the patient underwent exploratory laparotomy 18 years prior to our study. The operative note describes,

"The liver was massive and principally its substance was replaced by cysts. All cysts, measured from a few $\mathrm{mm}$ in diameter up to cysts that were $15-20 \mathrm{~cm}$ in their long diameter and possibly $8-10 \mathrm{~cm}$ in their transverse diameter ...

The left kidney had two cysts, each measuring $3-4 \mathrm{~cm}$ in diameter. The right kidney was completely depressed and rotated so that it assumed a transverse position. There was a very tiny cyst on the free portion of the right kidney. This measured $\frac{1}{2}-\frac{3}{4} \mathrm{~cm}$ in diameter. There were no visible cysts in the ovaries."

Two years later, she again underwent laparotomy. A considerable number of cysts were found throughout the entire liver parenchyma. Several small cysts were noted on each kidney; however, none seemed to be more than surface cysts. The patient still complained of intermittent pains in the abdomen. To date there has been no documentation of any renal abnormality.

\section{Cases III-I-III-4}

The four offspring of our patient, two males and two females, ages 24 to 35 , underwent ultrasound examination of the liver to document the possibility of cystic liver disease. None of the ultrasound examinations showed any cystic disease in the four offspring of our patient. Three of the four offspring (generation III) have children ranging from less than 1 year of age to 16 years of age. None of the generation IV members have had any documented complaints of gastrointestinal and/or renal ailments. They were not examined in this study. 


\section{Discussion}

Polycystic liver disease usually remains asymptomatic but is encountered at autopsy. In one series of 6141 autopsies, 11 cases had polycystic liver disease with the greatest frequency at 40 50 years of age and with a 3-8:1 female to male ratio (Poinso et al. 1952). Milutinovic et al. (1980) performed autopsy studies in patients with documented autosomal dominant polycystic kidney disease and found liver cysts in 46 $(29 \%)$ of 158 cases over 10 years of age. In their review, liver cysts were not identified in any of the patients at risk for polycystic kidney disease who did not present with renal cysts. In Milutinovic's et al. data, the extent of liver cystic disease in patients with autosomal dominant polycystic kidney disease correlated well with the extent of renal failure. In 65 patients, with creatinine clearance greater than $90 \mathrm{ml} / \mathrm{min}$, there was a $12 \%$ incidence of liver cysts while the incidence rose to $68 \%$ of 35 patients with creatinine clearance less than 10 $\mathrm{ml} / \mathrm{min}$. Dalgaard's (1952) extensive review of polycystic renal disease noted two relatives with clinically significant liver cysts without evident renal disease. Thus, isolated polycystic liver disease is a rare manifestation of polycystic renal disease. None of the patients in our family had polycystic kidney disease; none had abnormal kidney function.

The renal involvement in these patients is quite similar and minimal: each patient has a modest number of apparently simple cysts surrounded by normal cortex in normal sized kidneys. It is of interest that each patient had a rather large $3 \mathrm{~cm}$ cyst on the mid-lateral margin of the right kidney. The significance of this is unclear. The index case (II-2) demonstrated numerous $2-3 \mathrm{~mm}$ pancreatic cysts which could not be identified in her mother. As these cysts are very near the limits of resolution of our scanner, it may very well be that the mother has similar cysts which we are unable to detect. Neither patient had splenic involvement. The ovaries were not scanned.
Patients I-2 and II-2 differed markedly in the hepatic manifestations of this process. The mother has a modest number of small subcapsular cysts. The daughter, on the other hand, has a remarkable number of cysts which vary greatly in size. These cysts are more typical of polycystic liver disease than the mother's cysts (Fellman 1958). The distribution of the cysts is not subcapsular as in the mother. The liver cysts associated with polycystic kidney disease have been described as primarily subcapsular (Robbins 1967). The significance of the distribution of the cysts in these patients is unclear. The dilated biliary ducts in the daughter are probably an obstructive phenomenon due to the large hepatic cysts but the possibility of a congenital ectasia of these ducts cannot be excluded.

We believe the patients in our present study show hepatic polycystic disease independent of renal cystic disease. The pattern of genetic transmission of the liver cysts is consistent with an autosomal dominant pattern of inheritance. Sotaniemi et al. (1979) and Luoma et al. (1980) recently reported a family in which four of ten children of an affected mother had polycystic liver disease; this presentation is also consistent with an autosomal dominant pattern of inheritance. In these patients, there was no definition of extent of kidney involvement except for normal creatinine clearance. There is always the possibility that in the family members described by these authors kidney cysts were present which had not resulted in clinical disease. To our knowledge, our family is the second described supporting an autosomal dominant pattern of inheritance for polycystic liver disease, the family discussed above being the first. We believe our patients manifest liver cystic disease independent of significant renal cystic disease. Liver cysts associated with polycystic kidney disease do not cause liver enlargement and are rarely palpable clinically (Robbins 1967). None of our patients had bilateral renal cysts and their degree of renal cystic involvement is such as is to be found in 
many normal individuals. On the other hand, a $100 \%$ incidence of liver cysts among individuals with polycystic kidney disease has never been reported. There are several possible explanations for the lack of liver cysts in the offspring of our patients. The lack of cysts could be due to variable expressivity and/or penetrance. As all four members of the III generation are under 35 years of age, it is possible that they have not reached the age of full gene penetrance. Alternatively, none of these four individuals may have received the gene for polycystic liver disease from their mother.

\section{References}

Cairns, H. W. B. (1925). Heredity in polycystic disease of the kidneys. Quart. J. Med. 18, 359-370.

Dalgaard, O. Z. (1952). Bilateral polycystic disease of the kidneys. A follow-up of 284 patients and families. Acta med. scand. (Suppl.) 328, 1-255.

Davis, C. R. (1937). Non parasitic cysts of the liver. Amer. J. Surg. 35, 590-594.

Fellman, M. (1958). Polycystic disease of the liver. Amer. J. Gastroenterol. 29, 83-86.

Fittipaldi, C. \& G. De Chiaraa (1935). Non-parasitic cysts of the liver. Pathologica 27, 503-517.

Gardner, Jr., K. D. (1976). Cystic Diseases of the Kidney. New York, John Wiley \& Sons, pp. 202203.
Luoma, P. V., E. A. Sotaniemi \& C. Ehnholm (1980). Low high-density lipoprotein and reduced antipyrine metabolism in members of a family with polycystic liver disease. Scand. J. Gastroenterol. 15, 869-873.

Milutinovic, J., P. J. Fialkow, T. G. Rudd, L. Y. Agodoa, L. A. Phillips \& J. I. Bryant (1980). Liver cysts in patients with autosomal dominant polycystic kidney disease. Amer. J. Med. 68, 741-744.

Moschowitz, E. (1906). Non parasitic cysts (congenital) of the liver, with a study of aberrant bile ducts. Amer. J. med. Sci. 131, 674-699.

Poinso, R., H. Monges \& H. Payan (1952). La Maladie Kystique du Foie. Paris, Expansion Scientifique Francaise.

Robbins, S. L. (1967). Pathology, 3rd Ed. Philadelphia, W. B. Saunders, p. 901.

Sotaniemi, E. A., P. V. Luoma, P. M. Arvensivu \& K. A. Sotaniemi (1979). Impairment of drug metabolism in polycystic non-parasitic liver disease. Brit. J. clin. Pharmacol. 8, 331-335.

Williams, J. A. \& J. D. E. Price (1970). Liver cysts in end-stage uremia due to polycystic kidney disease. Canad. med. Assoc. J. 102, 856-857.

\section{Address:}

Robert P. Erickson, M.D.

Department of Human Genetics, Bor 015

The University of Michigan Medical School

1137 E. Catherine Street

Ann Arbor, MI 48109

U.S.A. 